Tecno Lógicas

ISSN 0123-7799

Vol. 19, No. 36, pp. 91-102

Enero-junio de 2016

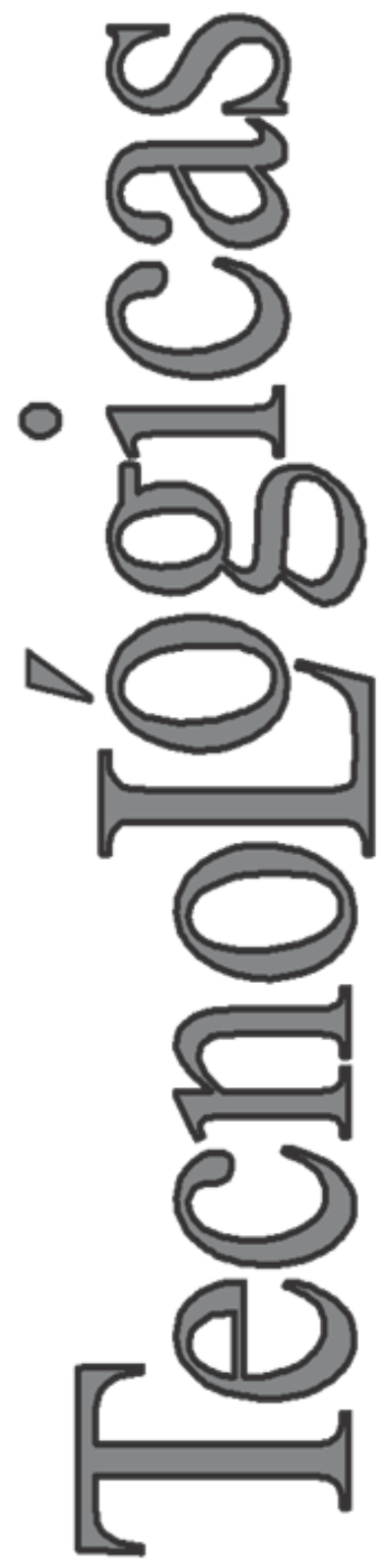

(C) Copyright 2015 por autores y Tecno Lógicas Este trabajo está licenciado bajo una Licencia Internacional Creative Commons Atribución (CC BY)

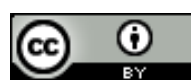

\section{Utilización de un residuo mineral industrial como catalizador en la hidroconversión de dibenciléter y de un carbón subbituminoso colombiano}

\author{
The use of an industrial mineral waste as \\ catalyst in the hydroconversion of \\ dibenzylether and a colombian \\ subbituminous coal
}

\author{
Juliana Sánchez-Castañeda ${ }^{1}$, Andrés Moreno-Lopera ${ }^{2}$ \\ y Wilson A. Ruiz-Machado ${ }^{3}$
}

Recibido: 9 de octubre de 2015, Aceptado: 30 de diciembre de 2015

Cómo citar / How to cite

J. Sánchez-Castañeda, A. Moreno-Lopera y W. A. Ruiz-Machado, "Utilización de un residuo mineral industrial como catalizador en la hidroconversión de dibenciléter y de un carbón subbituminoso colombiano”, Tecno Lógicas, vol. 19, no. 36, pp. 91-102, 2016.

1 MSc. en Ciencias Químicas, Grupo Química de Recursos Energéticos y Medio Ambiente, Instituto de Química, Facultad de Ciencias Exactas y Naturales, Universidad de Antioquia, Medellín-Colombia, juliana.sanchezc@udea.edu.co

2 PhD. en Ciencias Químicas, Grupo Química de Recursos Energéticos y Medio Ambiente, Instituto de Química, Facultad de Ciencias Exactas y Naturales, Universidad de Antioquia, Medellín-Colombia, amoreno.udea@gmail.com

3 PhD. en Ciencias Químicas, Grupo Química de Recursos Energéticos y Medio Ambiente, Instituto de Química, Facultad de Ciencias Exactas y Naturales, Universidad de Antioquia, Medellín-Colombia, wilson.ruiz@udea.edu.co 


\title{
Resumen
}

Un residuo mineral industrial, proveniente de la producción de sulfato de aluminio, se utilizó como precursor para la preparación de un catalizador de bajo costo, activo en reacciones de hidroconversión de dibenciléter, como compuesto modelo de las uniones C-O en el carbón, y de un carbón subbtituminoso colombiano. El residuo mineral y un óxido de hierro comercial (material de referencia) se sometieron a un proceso de sulfuración con el propósito de generar un sulfuro de hierro tipo pirrotita $\left(\mathrm{Fe}_{1-\mathrm{xS}} \mathrm{S}\right.$ ), el cual es la fase activa del hierro en reacciones de hidroconversión. Los sólidos se caracterizaron por difracción de rayos X, fluorescencia de rayos X, espectroscopía Raman y microscopía electrónica de barrido. Las pruebas de actividad catalítica, con el compuesto modelo y el carbón, se realizaron a $5 \mathrm{MPa} \mathrm{H}_{2}$, a temperaturas entre $240{ }^{\circ} \mathrm{C}$ y $400{ }^{\circ} \mathrm{C}$ y tiempos de reacción de 0,5 h y $1 \mathrm{~h}$. Los resultados mostraron que el residuo mineral industrial fue más activo que el material de referencia y que presentó alta actividad hacia la ruptura de los enlaces $\mathrm{C}-\mathrm{O}$ en el dibenciléter, siendo estos los enlaces responsables del ensamble de asfaltenos y preasfaltenos en la estructura molecular del carbón. Adicionalmente, la conversión del carbón incrementó significativamente en presencia del residuo mineral sulfurado alcanzando un valor de $83,9 \%$, valor 1,8 veces mayor que para la reacción sin catalizador, evidenciando el potencial del residuo mineral para ser usado como catalizador en la hidroconversión del carbón, debido a su buena actividad y bajo costo.

\section{Palabras clave}

Bauxita, sulfuros de hierro, dibenciléter, hidroconversión, carbón.

\begin{abstract}
An industrial mineral waste produced in the aluminum sulphate industry was selected as a low cost catalyst precursor. This material has been active in dibenzylether hydroconversion, as a model compound of $\mathrm{C}-\mathrm{O}$ linkages in coal. Besides, it was used in the hydroconversion of a colombian subbituminous coal. The mineral waste and a commercial iron oxide (as reference material) were sulfided in order to produce iron sulfide, pyrrhotite type $\left(\mathrm{Fe}_{1-\mathrm{x}} \mathrm{S}\right)$, which is the active phase in hydroconversion reactions. The solids were characterized by X-ray fluorescence, X-ray diffraction, Raman spectroscopy and scanning electron microscopy. Catalytic tests with the model compound and coal were carried out at $5 \mathrm{MPa}$ of $\mathrm{H}_{2}$, temperatures between $240{ }^{\circ} \mathrm{C}$ and $400{ }^{\circ} \mathrm{C}$ and reaction times were $0.5 \mathrm{~h}$ or $1 \mathrm{~h}$. The results showed a higher activity of the industrial mineral waste compared with the reference material in the dibenzylether $\mathrm{C}-\mathrm{O}$ bond cleavage. These kind of bonds are responsible for asphalthenes and preasphalthenes assembly in the molecular coal structure. In addition, coal conversion in presence of catalysts was $83.9 \%$, this value was 1.8 times higher than conversion without catalyst. Results show the potential of the industrial mineral waste to be used as catalyst in the hydroconversion of coal due to its good activity and low cost.
\end{abstract}

\section{Keywords}

Bauxite, iron sulfides, dibenzylether, hydroconversion, coal. 


\section{INTRODUCCIÓN}

La utilización de residuos, provenientes de los procesos de producción de alúmina o sulfato de aluminio a partir de bauxita ha sido ampliamente reportada en la literatura con el propósito de dar un valor agregado, por su difícil disposición y porque constituyen un problema ambiental, debido a la cantidad que se genera de ellos a nivel mundial [1]-[6]. Estos residuos se caracterizan por estar constituidos por óxidos de hierro, aluminio, silicio y titanio, por lo que muchos de los usos alternativos que se les ha dado aprovechan su composición [2]. Entre los usos principales se encuentran la recuperación de metales como hierro, aluminio y titanio [4], la obtención de cemento o acero y otros materiales para la construcción [1], y la obtención de catalizadores para procesos de oxidación [2]. Otra de las aplicaciones ha sido como materia prima para la preparación de catalizadores a base de sulfuros de hierro con actividad catalítica en el hidrocraqueo de fracciones pesadas de petróleo. Además, se ha encontrado que la presencia de óxidos de silicio y aluminio en este tipo de materiales tiene un efecto positivo en las reacciones de craqueo comparado con materiales compuestos solamente por óxidos de hierro [7], [8]. Otra de las aplicaciones de los residuos de bauxita es la licuefacción de carbón, en la que la fase activa es un sulfuro de hierro tipo pirrotita ( $\left.\mathrm{Fe}_{1-x} \mathrm{~S}\right)$ [9]. En este proceso los catalizadores más utilizados son los sulfuros de hierro provenientes de minerales como la pirita $\left(\mathrm{MoS}_{2}\right)$, o de procesos de sulfuración de óxidos de hierro comerciales $\mathrm{u}$ oxihidróxidos como la limonita ( $\alpha^{-}$ $\mathrm{FeOOH)}$ [10]. Los sulfuros de hierro promueven la pirolisis del carbón, favorecen la activación del hidrógeno molecular [11], [12] y presentan una mayor disponibilidad y menor costo comparado con otros metales de transición, lo cual es importante debido a que el catalizador queda mezclado con la fracción de sólidos y en ocasiones debe ser descartado.
La licuefacción de carbón es un proceso implementado comercialmente. Desde el año 2010 está en operación la planta construida en Shenhua (China), la cual tiene una capacidad aproximada de 3Mt/año [13]. El proceso se lleva a cabo a temperaturas entre $380-450^{\circ} \mathrm{C}$ y presiones de hidrógeno en el rango de 30-100 bar [10]. El propósito de la licuefacción es producir combustibles líquidos o compuestos químicos de interés a partir de carbón y disminuir la dependencia de las reservas de petróleo [10]. La producción de líquidos por licuefacción de carbón requiere la ruptura de su compleja estructura molecular, lo que involucra reacciones de hidrogenación, craqueo, eliminación de heteroátomos y apertura de anillos [8]. En general, se acepta que dicha ruptura se da en dos etapas: una etapa inicial en la que se producen asfaltenos y preasfaltenos, en la que las reacciones que priman son el rompimiento de enlaces $\mathrm{C}-\mathrm{C}$ y $\mathrm{C}-\mathrm{O}$ exocíclicos [14]; y una segunda etapa en la que los asfaltenos y preasfaltenos son transformados en una fracción de hidrocarburos líquidos mediante reacciones de hidrogenación de estructuras aromáticas, apertura de anillos y eliminación de heteroátomos [14]. En ocasiones, estas dos etapas se realizan de manera separada, siendo los objetivos de la primera etapa eliminar la materia mineral presente en el carbón, -a la cual se le atribuye la desactivación de los catalizadores- y generar preasfaltenos y asfaltenos que se solubilicen en solventes como tetrahidrofurano (THF) o piridina. Adicionalmente, en la primera etapa es adecuado utilizar catalizadores que se puedan descartar, ya que estos quedan mezclados con la fracción de carbón sin reaccionar y con la materia mineral del carbón; mientras que en la segunda etapa se parte de una materia prima más fácil de tratar y en presencia de catalizadores con mayor actividad para la hidrogenación de aromáticos y eliminación de heteroátomos [14].

El objetivo del presente trabajo fue la obtención de un catalizador de bajo costo a 
partir de un residuo del proceso de producción de sulfato de aluminio para ser usado en la hidroconversión de dibenciléter, como compuesto modelo de las uniones $\mathrm{C}^{-} \mathrm{O}$ exocíclicas en el carbón, y en la hidroconversión de un carbón subbituminoso colombiano.

\section{METOdología}

\subsection{Preparación y caracterización de catali- zadores}

Como precursores catalíticos se eligieron un residuo mineral (RA) proveniente de una industria colombiana de la producción de sulfato de aluminio a partir de bauxita y un óxido de hierro comercial $\left(\mathrm{Fe}_{2} \mathrm{O}_{3}\right)$ marca Sigma-Aldrich (99\%) como material de referencia. El residuo mineral (RA) se secó a $120^{\circ} \mathrm{C}$ por 12 horas, se maceró y se tamizó para obtener la fracción entre $75 \mu \mathrm{m}$ y $100 \mu \mathrm{m}$. Posteriormente, se calcinó en atmósfera de aire estático a $500^{\circ} \mathrm{C}$ durante $3 \mathrm{~h}$.

Los precursores catalíticos se sometieron a un proceso de sulfuración en un reactor tipo tanque agitado a alta presión marca Ogawa de $25 \mathrm{~mL}$, equipado con un sistema de agitación magnética, una línea de entrada de gases y otra de salida de gases. Inicialmente, al reactor se agregó el precursor catalítico; azufre elemental (Panreac, 99,5\%) en una relación molar $\mathrm{S} / \mathrm{Fe}=2$; tetralina (Merck, 98\%) como solvente e hidrógeno a $5 \mathrm{MPa}$ de presión. Posteriormente, se calentó a $400^{\circ} \mathrm{C}$ y se dejó a esta temperatura por $0,5 \mathrm{~h}$. Luego, el reactor se enfrió rápidamente y los sólidos sulfurados ( $\mathrm{S}-\mathrm{RA} ; \mathrm{S}-\mathrm{Fe}_{2} \mathrm{O}_{3}$ ) se lavaron con hexano, y se secaron en una estufa a $100^{\circ} \mathrm{C}$ durante $3 \mathrm{~h}$.

La caracterización de los precursores y los sólidos sulfurados se llevó a cabo por fluorescencia de rayos $\mathrm{X}(\mathrm{XRF})$ en un espectrómetro Thermo Optim'x, para determinar la composición química. Las fases cristalinas y la formación del sulfuro de hierro se determinaron por difracción de rayos $\mathrm{X}$ (XRD) en un difractómetro Panalytical X'PERT PRO MPD, equipado con ánodo de cobre $\left(\mathrm{Cu} \mathrm{K} \mathrm{K}_{\alpha 1}\right.$ de 1,5406 ̊̊), operado a $45 \mathrm{kV}$ y $40 \mathrm{~mA}$., a un tiempo de paso de 0,033s. Como técnicas complementarias para la caracterización estructural se utilizaron microscopía electrónica de barrido (SEM-EDX) en un microscopio electrónico JEOL JSM 6490, y espectroscopía Raman en un equipo Horiba Jobin Yvon, modelo Labram HR usando como fuente de excitación luz láser de $632,8 \mathrm{~nm}$. Adicionalmente, se determinó el área superficial de los precursores mediante adsorción de nitrógeno, aplicando el modelo BET. La adsorción de nitrógeno se realizó a la temperatura de ebullición del nitrógeno $\left(-196^{\circ} \mathrm{C}\right)$ en un equipo Micromeritics ASAP 2020. La medida de área superficial se realizó usando $100 \mathrm{mg}$ de los sólidos calcinados y previamente desgasificados a $120^{\circ} \mathrm{C}$ durante 2 horas en vacío. La acidez del residuo mineral industrial se determinó mediante análisis de desorción a temperatura programada de amoniaco (TPD-NH3), empleando un equipo Micromeritics Autochem II chemisorption analyzer.

\subsection{Hidroconversión de dibenciléter (DBE)}

Como una prueba de actividad de los catalizadores sulfurados se llevó a cabo la hidroconversión de dibenciléter (DBE) (Merck, 98\%) [15], [16]. En la elección de la molécula modelo se tuvo en cuenta la presencia de grupos éter de alta reactividad en el carbón a los cuales se les atribuye las reacciones de depolimerización del mismo [15], siendo el DBE una de las moléculas presentes en los modelos moleculares de carbones subbituminosos [17].

Las reacciones se llevaron a cabo en el mismo reactor tipo batch en el que se realizaron las sulfuraciones. Las condiciones de reacción fueron: $5 \mathrm{MPa}$ de presión de hidrógeno en frío, temperaturas de $240^{\circ} \mathrm{C}, 320^{\circ} \mathrm{C}$ y $400^{\circ} \mathrm{C}$, con un tiempo de reacción de $0,5 \mathrm{~h}$. El dibenciléter se utilizó al 10\% usando como solvente tetralina, debido a que este 
solvente es un compuesto hidroaromático que actúa como agente de transferencia de hidrógeno en reacciones de hidroconversión [16]. El precursor del catalizador (residuo mineral $u$ óxido de hierro comercia) se utilizó al 5\% respecto al dibenciléter y la sulfuración se realizó in-situ usando azufre elemental [11]. Los productos líquidos de las reacciones se caracterizaron por GCFID-MS usando un equipo marca Shimadzu 2010 Plus equipado con una columna capilar SHRXI - 5MS (5\% fenil 95\% metilpolisiloxano) marca Shidmadzu de $30 \mathrm{~m} \mathrm{x}$ $0,25 \mathrm{~mm} \times 0,25 \mu \mathrm{m}$. Se utilizó una relación split de 30 y un volumen de inyección de $0,5 \mu \mathrm{L}$. La temperatura inicial del horno fue de $50^{\circ} \mathrm{C}$ y se calentó hasta $200^{\circ} \mathrm{C}$ a una velocidad de $10^{\circ} \mathrm{C} / \mathrm{min}$ y se mantuvo a esta temperatura durante $5 \mathrm{~min}$.

\subsection{Reacciones de hidroconversión de car- bón}

Se seleccionó un carbón subbituminoso colombiano (Titiribí, Antioquia) con un tamaño de partícula menor a $147 \mu \mathrm{m}$, el cual tiene una composición determinada por análisis elemental de $74,3 \% \mathrm{C}, 5,2 \% \mathrm{H}$, $5,3 \%$ N, 0,8\% S y 14,3\% O (por diferencia). Y presenta un porcentaje de volátiles de $37 \%$, un porcentaje de carbono fijo de $58 \%$ y un porcentaje de cenizas de $5 \%$, determinados por análisis termogravimétrico. Los experimentos se realizaron a $5 \mathrm{MPa}$ de presión de hidrógeno a $400{ }^{\circ} \mathrm{C}$ durante $1 \mathrm{~h}$. Para la reacción se utilizó una relación tetralina: carbón de $4: 1$, el catalizador al $10 \%$ respecto al carbón y sulfuración insitu del precursor catalítico.

Se realizó una extracción con tetrahidrofurano (THF) para separar los productos (solubles en THF) del residuo (insolubles en THF). Ambas fracciones se caracterizaron por análisis termogravimétrico en un equipo TA Instruments Q500 y por análisis elemental (CHNO) en un equipo TruSpec CHNS-O.

\section{RESULTADOS Y DISCUSIÓN}

\subsection{Caracterización de los precursores catalíticos}

En la Tabla 1 se presentan los resultados de composición química de los precursores catalíticos. Para el precursor RA la composición se determinó por XRF y para el óxido de hierro comercial se reportan los valores de acuerdo con las especificaciones del reactivo. Se puede observar que el residuo RA presenta un porcentaje de hierro de $8,8 \%$ expresado como óxido, y un alto contenido de óxidos de silicio y aluminio de $55,8 \%$ y $27,1 \%$ respectivamente. Los valores de composición de RA están de acuerdo con los valores reportados en la literatura para materiales similares tipo bauxita $\mathrm{y}$ barro rojo [18]. Es de notar, que la presencia de óxidos de aluminio y de silicio en el residuo, a diferencia del material de referencia que contiene solo óxido de hierro, genera mayores áreas superficiales (107 $\mathrm{m}^{2} / \mathrm{g}$ para RA comparado con $5 \mathrm{~m}^{2} / \mathrm{g}$ para $\mathrm{Fe}_{2} \mathrm{O}_{3}$ ) y acidez cuyo valor determinado por TPD de $\mathrm{NH}_{3}$ es de $276 \mu \mathrm{mol} / \mathrm{g}$, la cual es comparable con la acidez de materiales del tipo sílico alúminas usados en reacciones de hidrocraqueo, con valores entre 160 $\mu \mathrm{mol} / \mathrm{g}$ y $540 \mu \mathrm{mol} / \mathrm{g}$ [19]. De acuerdo con este valor de acidez se puede esperar que el material favorezca reacciones de craqueo de la molécula modelo y del carbón, además de propiciar reacciones de hidrogenación atribuidas al sulfuro de hierro, lo que puede significar una mejora en la conversión.

En la Fig. 1 (A y B) se observan los patrones de difracción de rayos $\mathrm{X}$ para los precursores catalíticos. Para la muestra $\mathrm{Fe}_{2} \mathrm{O}_{3}$ se presentan picos a valores de $2 \theta$ de $24,1^{\circ}, 33,1^{\circ}, 35,6^{\circ}, 40,8^{\circ}, 49,4^{\circ}, 59,0^{\circ}, 62,3^{\circ} \mathrm{y}$ $63,9^{\circ}$, característicos de la fase hematita [20]. Para la muestra RA se observan además de las señales de hematita, señales características de cuarzo estando ubicada la principal a $26,8^{\circ}$ y de gibbsita a $20,9^{\circ}$ y $21,9^{\circ}$ [21]. 
Tabla 1. Propiedades de los precursores catalíticos. Fuente: Autores

\begin{tabular}{ccc}
\hline \multirow{2}{*}{ Propiedad } & \multicolumn{2}{c}{ Material } \\
& $\mathrm{Fe}_{2} \mathrm{O}_{3}$ & $\mathrm{RA}$ \\
\hline Composición (\%) & & \\
$\mathrm{Al}_{2} \mathrm{O}_{3}$ & -- & 27,1 \\
$\mathrm{SiO}_{2}$ & -- & 55,8 \\
$\mathrm{Fe}_{2} \mathrm{O}_{3}$ & 99,0 & 8,8 \\
$\mathrm{MoO}_{3}$ & -- & 0,1 \\
$\mathrm{SO}_{3}$ & -- & 3,3 \\
$\mathrm{TiO}_{2}$ & -- & 3,9 \\
$\mathrm{Otros}$ & 1,0 & 1,0 \\
Área superficial $\left(\mathrm{m}^{2} / \mathrm{g}\right)$ & 5 & 107 \\
Acidez $(\mu \mathrm{mol} / \mathrm{g})$ & N.D. & 276 \\
\hline
\end{tabular}

N.D. No determinado
En la Fig. 2 se encuentran los espectros Raman para los precursores catalíticos. Para el precursor $\mathrm{Fe}_{2} \mathrm{O}_{3}$ se identificó la fase hematita, lo cual es coherente con los resultados de XRD, con señales a $227 \mathrm{~cm}^{-1}$, $247 \mathrm{~cm}^{-1}, 293 \mathrm{~cm}^{-1}, 413 \mathrm{~cm}^{-1}$ y $613 \mathrm{~cm}^{-1}$ [22]. Similarmente, el espectro Raman de RA presenta bandas que se pueden asignar a hematita con valores de número de onda similares a los mencionados para $\mathrm{Fe}_{2} \mathrm{O}_{3}$ y además no se descarta la presencia de algunas bandas correspondientes a gibbsita que pueden estar solapadas con las de hematita [22].

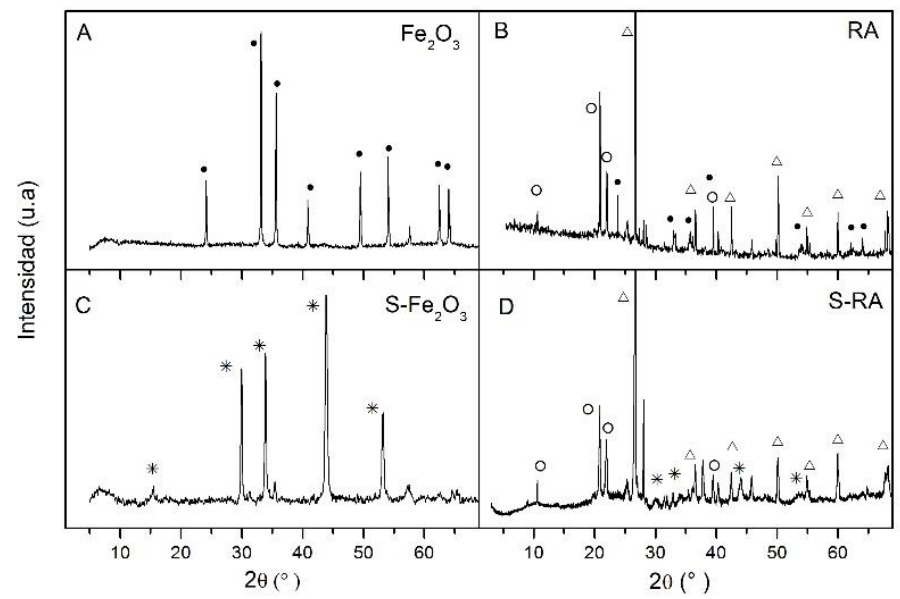

Fig. 1. Patrones de XRD: A) y B) precursores catalíticos. Señales para: (•) Hematita; (o) Gibbsita; ( $\Delta$ ) Cuarzo. C) y D) sólidos sulfurados. Señales para: (*) Pirrotita. Fuente: Autores

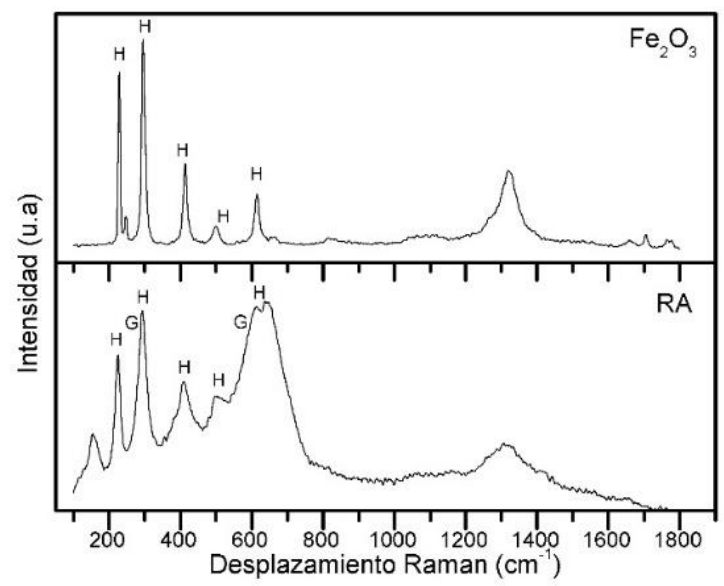

Fig. 2. Espectros Raman de los precursores catalíticos. Fuente: Autores 
Utilización de un residuo mineral industrial como catalizador en la hidroconversión de dibenciléter y de un carbón subbituminoso colombiano

\subsection{Caracterización de los sólidos sulfura- dos}

En la Fig. 1 (C y D) se presentan además los difractogramas de los sólidos sulfurados $\mathrm{S}-\mathrm{Fe}_{2} \mathrm{O}_{3}$ y $\mathrm{S}-\mathrm{RA}$. De la Figura se observan señales a valores de $2 \theta$ de $15,1^{\circ}$, $29,9^{\circ}, 33,8^{\circ}, 43,7^{\circ}, 53,11^{\circ}$ y $57,2^{\circ}$, las cuales corresponden a un sulfuro de hierro tipo pirrotita $\left(\mathrm{Fe}_{1-x} \mathrm{~S}\right)$, cuyos valores de $(1-\mathrm{x})$ se encuentran entre $0,875\left(\mathrm{Fe}_{7} \mathrm{~S}_{8}\right)$ y $1(\mathrm{FeS})$. La fase pirrotita se ha reportado en la literatura como la fase activa de los catalizadores a base de hierro en reacciones de hidroconversión [9].

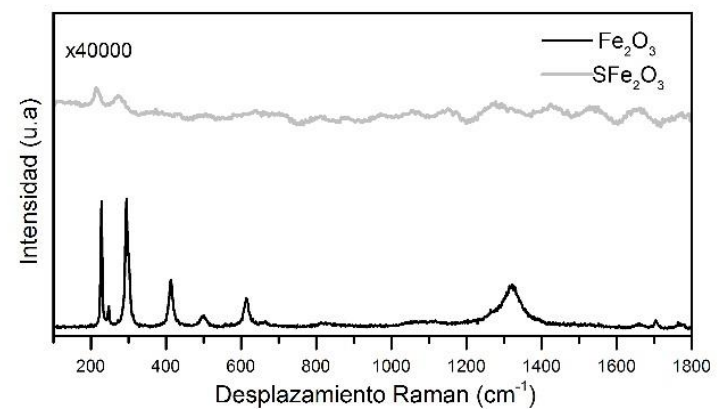

Fig. 3. Espectros Raman del precursor $\mathrm{Fe}_{2} \mathrm{O}_{3}$ y el sólido sulfurado $\mathrm{S}-\mathrm{Fe}_{2} \mathrm{O}_{3}$. Fuente: Autores

Como técnicas complementarias a la caracterización por XRD, los catalizadores sulfurados se analizaron además por espectroscopía Raman y SEM-EDX. En la Fig. 3 se comparan los espectros Raman del precursor $\mathrm{Fe}_{2} \mathrm{O}_{3}$ y el correspondiente sólido sulfurado. En ella se observa una disminución de cinco órdenes de magnitud en la intensidad de las bandas correspondientes a la hematita, siendo esto una evidencia de la transformación de los óxidos en sulfuros. Similarmente, se observó esta disminución en las señales para la muestra S-RA.

Por otra parte, mediante SEM-EDX se calcularon las relaciones atómicas $\mathrm{S} / \mathrm{Fe}$ para los precursores y sólidos sulfurados, las cuales se encuentran reportadas en la Tabla 2. De la misma se puede observar que luego del proceso de sulfuración la relación $\mathrm{S} / \mathrm{Fe}$ incrementa a valores cerca- nos a 1 , lo cual está de acuerdo con la fase pirrotita, identificada por XRD.

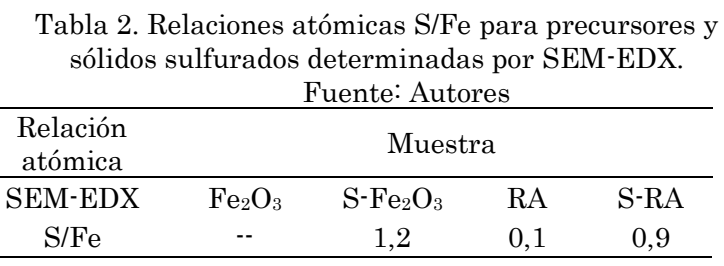

Adicionalmente, por SEM-EDX se realizó un mapeo elemental para las muestras $\mathrm{S}-\mathrm{Fe}_{2} \mathrm{O}_{3}$ y $\mathrm{S}-\mathrm{RA}$, con el propósito de observar la distribución de algunos elementos en varias regiones de las muestras. En la Fig. 4 se presenta el mapeo elemental (Fe, S, O) para la muestra sulfurada S$\mathrm{Fe}_{2} \mathrm{O}_{3}$, del cual se observa una alta concentración de hierro y azufre en las partículas analizadas y una concentración mucho menor de oxígeno comparado con los otros dos elementos, lo que se atribuye al proceso de sulfuración. Es de notar que la distribución de los tres elementos es homogénea en la región analizada.

En la Fig. 5 se puede observar que la muestra S-RA presenta una distribución homogénea del hierro y del azufre en varias regiones analizadas de la muestra, y se observa una coincidencia en los mapeos de ambos elementos, lo que evidencia la transformación del óxido de hierro en sulfuro de hierro $\left(\mathrm{Fe}_{1-x} \mathrm{~S}\right)$. Además, el aluminio y el silicio están en mayor concentración que el hierro y el azufre, lo cual podría tener un efecto positivo como soporte al que se le atribuyen ciertas funciones como la de incrementar la actividad catalítica por modificaciones estructurales, por dispersión de la fase activa y por introducir un carácter bifuncional al catalizador [23], [24]. La bifuncionalidad del catalizador se puede relacionar con la acidez encontrada para el material (Tabla 1). Sin embargo, se requiere un estudio más detallado acerca de la importancia del soporte en la aplicación como precursor catalítico para este tipo de residuos. 

subbituminoso colombiano
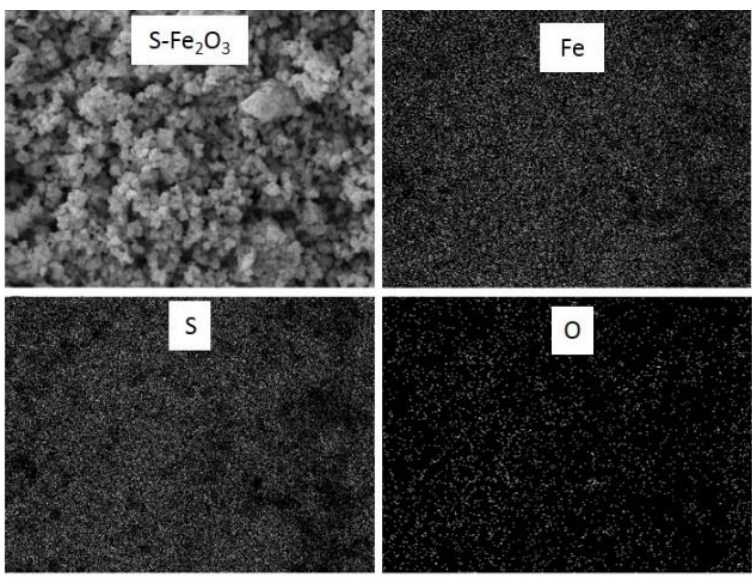

Fig. 4. Mapeo elemental (Fe, S, O) por SEM-EDX para S$\mathrm{Fe}_{2} \mathrm{O}_{3}$. Fuente: Autores
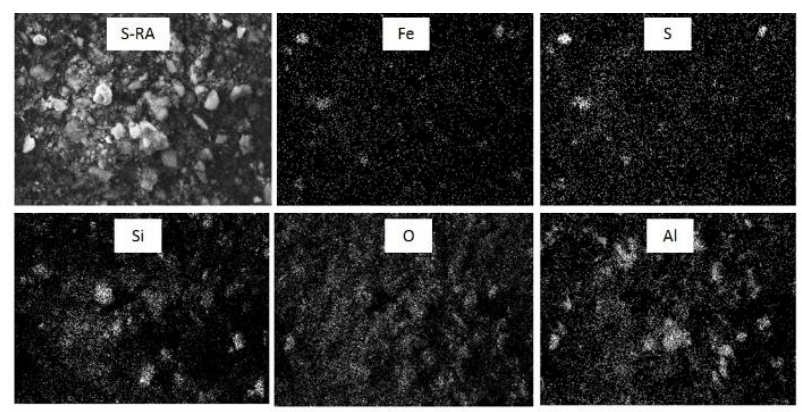

Figura 5. Mapeo elemental (Fe, S, O, Si, Al) por SEM-EDX para S-RA. Fuente: Autores

\subsection{Hidroconversión de dibenciléter (DBE)}

En la Fig. 6 se presentan los resultados de conversión y la distribución de los productos de craqueo para las diferentes reacciones con DBE. Los resultados muestran que la presencia de catalizador causó un incremento significativo en la conversión del dibenciléter. Para el catalizador S-RA la conversión a $240^{\circ} \mathrm{C}$ fue $66,4 \%$, mientras que en ausencia de catalizador fue $3,4 \%$. A $320^{\circ} \mathrm{C}$ se logra una conversión de $98,4 \%$ con S-RA, la cual es considerablemente mayor que el grado de conversión obtenido en ausencia de catalizador (18,3\%). Finalmente, la conversión a $400^{\circ} \mathrm{C}$ en presencia de S-RA fue $99,9 \%$ mientras que para la reacción no catalítica la conversión fue $55,0 \%$. Este valor para la conversión térmica a $400^{\circ} \mathrm{C}$ durante 30 min es comparable con el valor de $94,0 \%$ reportado en la lite- ratura a $400^{\circ} \mathrm{C}$ en presencia de tetralina y durante el doble del tiempo (60 min) [25].

Es de notar que los catalizadores S$\mathrm{Fe}_{2} \mathrm{O}_{3}$ y S-RA presentaron valores similares de conversión, excepto a $240{ }^{\circ} \mathrm{C}$, donde se observa una conversión mayor para $\mathrm{S}^{-}$ RA $(66,4 \%)$ comparado con la conversión de $\mathrm{S}-\mathrm{Fe}_{2} \mathrm{O}_{3}$ (58,1\%). Sin embargo, hay que tener presente que la masa total de precursor se mantuvo constante en todas las pruebas catalíticas, por lo que las reacciones con $\mathrm{S}-\mathrm{Fe}_{2} \mathrm{O}_{3}$ se realizaron en presencia de una mayor cantidad de fase activa ( $\mathrm{pi}^{-}$ rrotita), comparado con las reacciones con S-RA en la que además de óxido de hierro se tienen óxidos de silicio y aluminio.

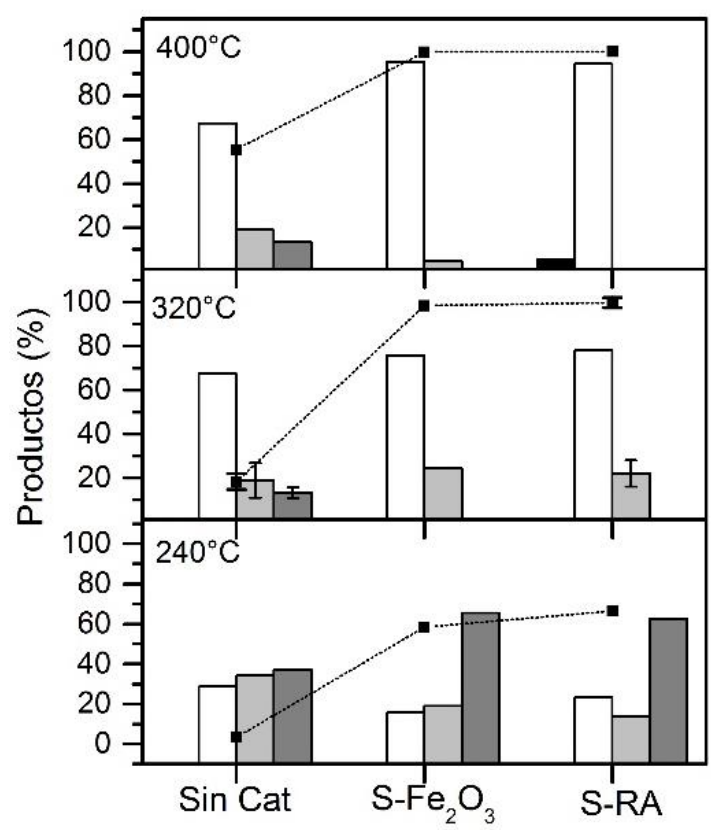

Fig. 6. Conversión y distribución de productos de craqueo para la hidroconversión de DBE a $240^{\circ} \mathrm{C}, 320^{\circ} \mathrm{C}$ y $400^{\circ} \mathrm{C}$. Conversión (línea punteada); Selectividades: $\mathbf{a}$ benceno; $\square$ tolueno; benzaldehído; alcohol bencílico. Fuente: Autores

La mayor actividad del catalizador SRA puede estar asociada principalmente con el efecto de la presencia del óxido de aluminio, el cual además de tener un efecto como soporte y favorecer la dispersión del sulfuro de hierro, le confiere al catalizador una funcionalidad ácida (de acuerdo con el valor de acidez del sólido $\mathrm{RA}$ reportado en la Tabla 1), la cual tiene un efecto positivo 
en las reacciones de craqueo del compuesto modelo, con respecto al catalizador de referencia que solo está compuesto por sulfuro de hierro cuya actividad es principalmente relacionada con las reacciones de hidrogenación [23].

Los productos de reacción encontrados para el dibenciléter están de acuerdo con los reportados en la literatura para este mismo compuesto modelo [16], [26]. Se identificaron benceno, tolueno, benzaldehído y alcohol bencílico como productos de craqueo. De acuerdo con los esquemas de reacción reportados en la literatura [16], [26], la formación de estos productos se da por ruptura homolítica del enlace C-O mediante lo cual se producen radicales bencilo, con los que se puede generar tolueno o benceno, y radicales benzoilo, a partir de los cuales se da la formación de benzaldehído o alcohol bencílico por donación o aceptación de un radical hidrógeno respectivamente. También se observaron algunos productos de recombinación con la tetralina (benciltetralinas), cuya formación se ha reportado en la literatura, cuando se utiliza este solvente donador de hidrógeno [16].

Con respecto a la distribución de productos, en la Fig. 6 se observa una disminución de los productos oxigenados (benzaldehído y alcohol bencílico) con el incremento en la temperatura, y esta disminución es mayor en presencia de los catalizadores. Es de resaltar que a $400{ }^{\circ} \mathrm{C}$ con el catalizador S-RA no se observan compuestos oxigenados. Lo anterior, evidencia la actividad del catalizador para la ruptura de enlaces $\mathrm{C}-\mathrm{O}$ exocíclicos y para la eliminación del oxígeno de estructuras del tipo éter, aldehído y alcohol. Por otro lado, este catalizador no presentó actividad para las reacciones de hidrogenación de estructuras aromáticas (ausencia de ciclohexano entre los productos), por lo cual se puede inferir que el catalizador S-RA podría presentar actividad en la producción de asfaltenos y preasfaltenos a partir del carbón [27], [14].

\subsection{Reacciones de hidroconversión de car- bón}

En la Tabla 3 se presentan los resultados de hidroconversión del carbón. Para la reacción sin catalizador el porcentaje de conversión fue $47,1 \%$. Por su parte, con el catalizador S-RA se obtuvo una conversión de $83,9 \%$, valor 1,8 veces mayor a la conversión de la reacción no catalítica. El incremento en la conversión del carbón, a compuestos de menor peso molecular en presencia del catalizador, está de acuerdo con los resultados de hidroconversión de DBE. En la literatura se encuentran reportados porcentajes de conversión de carbón entre 40 y $80 \%$ cuando el proceso se realiza a $400^{\circ} \mathrm{C}$ a presiones entre $50-170$ bar $\mathrm{H}_{2}$ con tiempos de reacción entre 30 y $60 \mathrm{mi}^{-}$ nutos y en presencia de catalizadores a base de sulfuro de hierro [28]-[30], lo que indica que en la presente investigación se obtuvieron altas conversiones en presencia del catalizador a base del residuo mineral industrial $\mathrm{y}$ a presiones relativamente bajas de hidrógeno lo que podría hacer más viable el proceso.

La fracción de solubles en THF contiene preasfaltenos asfaltenos y líquidos. Sin embargo, mediante extracciones con hexano, se encontró que el rendimiento a líquidos fue menor a 5\%, lo que indica que los productos principales fueron asfaltenos y preasfaltenos. Este resultado está de acuerdo con la alta actividad del catalizador S-RA para la ruptura de enlaces C-O y con la baja actividad para la hidrogenación de estructuras aromáticas. En este sentido, reportes de la literatura indican que la producción de líquidos requiere reacciones de hidrogenación de estructuras aromáticas, apertura de anillos y eliminación de heteroátomos, por lo que se requieren catalizadores altamente activos para la hidrogenación [14], [15], [31]. 
Tabla 3. Conversión y caracterización de productos de la licuefacción de carbón. Fuente: Autores

\begin{tabular}{|c|c|c|c|c|c|}
\hline & 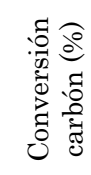 & 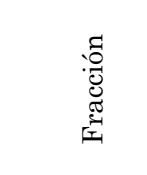 & 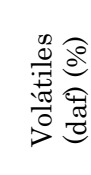 & 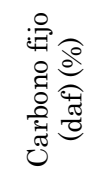 & 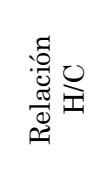 \\
\hline Carbón & -- & -- & 39,1 & 60,9 & 0,86 \\
\hline \multirow{2}{*}{ Sin Cat } & \multirow{2}{*}{47,1} & Productos & 53,6 & 46,4 & 0,94 \\
\hline & & Residuo & 41,7 & 58,3 & 0,85 \\
\hline \multirow{2}{*}{ S-RA } & \multirow{2}{*}{83,9} & Productos & 56,3 & 43,6 & 0,96 \\
\hline & & Residuo & 40,3 & 59,7 & 0,84 \\
\hline
\end{tabular}

En la Tabla 3 también se reporta la caracterización de las fracciones de productos y residuos y se compara con las propiedades del carbón. Se observa un incremento en el contenido de volátiles en los productos con respecto al carbón, con valores de $53,6 \%$ para la reacción no catalítica y $56,3 \%$ para la reacción con $\mathrm{S}-\mathrm{RA}$, comparado con $39,1 \%$ para el carbón. Es de anotar que el incremento en la cantidad de volátiles sugiere la presencia de fragmentos más pequeños y con menor grado de condensación. Adicionalmente, el incremento en la relación $\mathrm{H} / \mathrm{C}$ de 0,86 para el carbón a 0,95 para los productos, evidencia la presencia de moléculas con un mayor grado de hidrogenación y menor condensación que en el carbón. Relaciones H/C con cercanas a 0,95 han sido reportadas previamente para asfaltenos y preasfaltenos derivados de carbones subbtimuninosos [10].

\section{CONCLUSIONES}

La caracterización de los precursores y los sólidos sulfurados indica que el proceso de sulfuración fue efectivo, generándose un sulfuro de hierro tipo pirrotita $\left(\mathrm{Fe}_{1 \times \mathrm{x}} \mathrm{S}\right)$, tanto con el material de referencia como con el residuo mineral industrial. Adicionalmente, la presencia de óxido de aluminio en el residuo puede estar teniendo un efecto positivo como soporte, generando acidez y favoreciendo las reacciones de craqueo. Lo anterior se vio reflejado en la mayor actividad del catalizador proveniente del residuo comparado con el material de referencia.

Por otro lado, los materiales sulfurados fueron altamente activos en la hidroconversión del dibenciléter, como compuesto modelo de las uniones éter presentes en el carbón. Estos grupos funcionales tipo éter son los responsables del ensamble de asfaltenos y preasfaltenos para formar la es$^{-}$ tructura molecular del carbón. En este sentido, el catalizador presentó actividad para la hidroconversión de un carbón subbituminoso con una conversión del 83,4\%, obteniendo como productos principales asfaltenos y preasfaltenos, los cuales constituyen una materia prima más fácil de tratar en procesos posteriores para la obtención de líquidos. Lo anterior evidencia la potencialidad del residuo mineral industrial para ser usado como catalizador en un proceso en dos etapas debido a su buena actividad y bajo costo.

\section{AGRADECIMIENTOS}

Los autores agradecen a COLCIENCIAS por la financiación del proyecto código: 1115-521-28810 y a la Universidad de Antioquia por el Programa de Sostenibilidad.

\section{REFERENCIAS}

[1] W. Liu, X. Chen, W. Li, Y. Yu, and K. Yan, "Environmental assessment, management and utilization of red mud in China," J. Clean. Prod., vol. 84, pp. 606-610, Dec. 2014.

[2] S. C. Kim, S. W. Nahm, and Y.-K. Park, "Property and performance of red mud-based catalysts for the complete oxidation of volatile organic compounds," J. Hazard. Mater., vol. 300, pp. 104-113, Dec. 2015. 
[3] Y. Huang, G. Han, J. Liu, and W. Wang, "A facile disposal of Bayer red mud based on selective flocculation desliming with organic humics," $J$. Hazard. Mater., vol. 301, pp. 46-55, Jan. 2016.

[4] Y. Liu and R. Naidu, "Hidden values in bauxite residue (red mud): Recovery of metals," Waste Manag., vol. 34, no. 12, pp. 2662-2673, Dec. 2014.

[5] G. Power, M. Gräfe, and C. Klauber, "Bauxite residue issues: I. Current management, disposal and storage practices," Hydrometallurgy, vol. 108, no. 1-2, pp. 33-45, Jun. 2011.

[6] C. Klauber, M. Gräfe, and G. Power, "Bauxite residue issues: II. options for residue utilization," Hydrometallurgy, vol. 108, no. 1-2, pp. 11-32, Jun. 2011.

[7] A. Bhattacharyya and B. J. Mezza, "patente process for using iron oxide and alumina for slurry hydrocracking.pdf." 2012.

[8] J.-H. Lv, X.-Y. Wei, Y.-H. Wang, L.C. Yu, D.-D. Zhang, X.-M. Yue, T.-M. Wang, J. Liu, Z.-M. Zong, X. Fan, and Y.-P. Zhao, "Light fraction from catalytic hydroconversion of two Chinese coals in cyclohexane over a solid acid," Fuel Process. Technol., vol. 129, pp. 162-167, Jan. 2015.

[9] N. Shah, J. Zhao, F. E. Huggins, and G. P. Huffman, "In Situ XAFS Spectroscopic Studies of Direct Coal Liquefaction Catalysts," Energy \& Fuels, vol. 10, no. 2, pp. 417-420, Jan. 1996.

[10] S. Vasireddy, B. Morreale, A. Cugini, C. Song, and J. J. Spivey, "Clean liquid fuels from direct coal liquefaction: chemistry, catalysis, technological status and challenges," Energy Environ. Sci., vol. 4, no. 2, pp. 311-345, 2011.

[11] T. Kaneko, K. Tazawa, T. Koyama, K. Satou, K. Shimasaki, and Y. Kageyama, "Transformation of Iron
Catalyst to the Active Phase in Coal Liquefaction," Energy \& Fuels, vol. 12, no. 5, pp. 897-904, Sep. 1998.

[12] D.-D. Zhang, Z.-M. Zong, J. Liu, Y.H. Wang, L.-C. Yu, J.-H. Lv, T.-M. Wang, X.-Y. Wei, Z.-H. Wei, and Y. $\mathrm{Li}$, "Catalytic hydroconversion of Geting bituminous coal over FeNiS/Y-Al2O3," Fuel Process. Technol., vol. 133, pp. 195-201, May 2015.

[13] Z. Liu, S. Shi, and Y. Li, "Coal liquefaction technologiesDevelopment in China and challenges in chemical reaction engineering," Chem. Eng. Sci., vol. 65 , no. 1, pp. 12-17, Jan. 2010.

[14] Z. Wang, H. Shui, Z. Lei, S. Ren, S. Kang, H. Zhou, X. Gu, and J. Gao, "Study of the preasphaltenes of coal liquefaction and its hydro-conversion kinetics catalyzed by $\mathrm{SO} 42-/ \mathrm{ZrO} 2$, Fuel Process. Technol., vol. 92, no. 10, pp. 1830-1835, Oct. 2011.

[15] I. Merdrignac and A. Quignard, "Hydroconversion of coals," in Catalysis by transition metal sulphides: From molecular theory to industrial application, 2013, pp. 758-776.

[16] N. Ikenaga, Y. Kobayashi, S. Saeki, T. Sakota, Y. Watanabe, H. Yamada, and T. Suzuki, "Hydrogen-Transfer Reaction of Coal Model Compounds in Tetralin with Dispersed Catalysts," Energy \& Fuels, vol. 8, no. 4, pp. 947-952, Jul. 1994.

[17] J. P. Mathews and A. L. Chaffee, "The molecular representations of coal - A review," Fuel, vol. 96, pp. 114, Jun. 2012.

[18] S. Sushil and V. S. Batra, "Catalytic applications of red mud, an aluminium industry waste: A review," Appl. Catal. B Environ., vol. 81, no. 1-2, pp. 64-77, May 2008.

[19] M. K. Mardkhe, K. Keyvanloo, C. H. Bartholomew, W. C. Hecker, T. M. Alam, and B. F. Woodfield, "Acid site properties of thermally stable, silica- 
doped alumina as a function of silica/alumina ratio and calcination temperature," Appl. Catal. A Gen., vol. 482, pp. 16-23, Jul. 2014.

[20] M. P. Pomiès, G. Morin, and C. Vignaud, "XRD study of the goethitehematite transformation: Application to the identification of heated prehistoric pigments," Eur. J. Solid State Inorg. Chem., vol. 35, no. 1, pp. 9-25, Jan. 1998.

[21] A. Malki, Z. Mekhalif, S. Detriche, G. Fonder, A. Boumaza, and A. Djelloul, "Calcination products of gibbsite studied by X-ray diffraction, XPS and solid-state NMR," J. Solid State Chem., vol. 215, pp. 8-15, Jul. 2014.

[22] D. L. A. de Faria, S. Venâncio Silva, and M. T. de Oliveira, "Raman microspectroscopy of some iron oxides and oxyhydroxides," $J$. Raman Spectrosc., vol. 28, no. 11, pp. 873-878, Nov. 1997.

[23] T. K. T. Ninh, L. Massin, D. Laurenti, and M. Vrinat, "A new approach in the evaluation of the support effect for NiMo hydrodesulfurization catalysts," Appl. Catal. A Gen., vol. 407, no. 12, pp. 29-39, Nov. 2011.

[24] C.-E. Hédoire, C. Louis, A. Davidson, M. Breysse, F. Maugé, and M. Vrinat, "Support effect in hydrotreating catalysts: hydrogenation properties of molybdenum sulfide supported on $B^{-}$ zeolites of various acidities," $J$. Catal., vol. 220, no. 2, pp. 433-441, Dec. 2003.
[25] J. J. Vlieger, "Aspects of the chemistry of hydrogen donor solvent coal liquefaction," Universidad de Tecnologíca de Delft, 1988.

[26] K. Chiba, H. Tagaya, T. Yamauchi, and S. Sato, "Effect of solvents on thermal cracking of model compounds typical of coal," Ind. Eng. Chem. Res., vol. 30, no. 6, pp. 11411145, Jun. 1991.

[27] T. Gauthier, P. Danialfortain, I. Merdrignac, I. Guibard, and A. Quoineaud, "Studies on the evolution of asphaltene structure during hydroconversion of petroleum residues," Catal. Today, vol. 130, no. 2-4, pp. 429-438, Jan. 2008.

[28] X. Li, H. Hu, L. Jin, S. Hu, and B. $\mathrm{Wu}$, "Approach for promoting liquid yield in direct liquefaction of Shenhua coal," Fuel Process. Technol., vol. 89, no. 11, pp. 10901095, Nov. 2008.

[29] H. Hu, G. Sha, and G. Chen, "Effect of solvent swelling on liquefaction of Xinglong coal at less severe conditions," Fuel Process. Technol., vol. 68 , no. 1 , pp. 33-43, Oct. 2000.

[30] I. de Marco Rodriguez, M. J. Chomón, B. Caballero, P. L. Arias, and J. A. Legarreta, "Liquefaction behaviour of a Spanish subbituminous A coal under different conditions of hydrogen availability," Fuel Process. Technol., vol. 58, no. 1, pp. 17-24, Nov. 1998.

[31] I. Ceyhun, "Kinetic Studies on Karliova Coal 1," Theor. Found. Chem. Eng., vol. 37, no. 4, pp. 416420, 2003. 